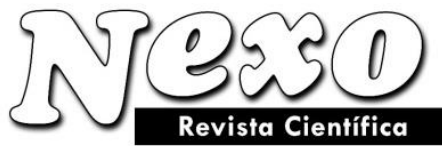

Universidad Nacional de Ingeniería COPYRIGHT @ (UNI). TODOS LOS DERECHOS RESERVADOS http://revistas.uni.edu.ni/index.php/Nexo https://doi.org/10.5377/nexo.v34i05.13099

\title{
Concreto reciclado a partir de escombros de mampostería de bloque de cemento
}

\section{Recycled concrete from cement block masonry debris}

\author{
Erasmo José Aguilar Arriola ${ }^{1, *}$, Eddisson Francisco Hernández ${ }^{2}$, Pablo Antonio Espinoza ${ }^{3}$ \\ ${ }^{1}$ Universidad Nacional de Ingeniería. Facultad de Arquitectura. Managua, Nicaragua. \\ * erasmo.aguilar@farq.uni.edu.ni \\ 0000-0002-6267-9405 (https://orcid.org/0000-0002-6267-9405) \\ ${ }^{2}$ Universidad Nacional de Ingeniería. Facultad de Arquitectura. Managua, Nicaragua. \\ *eddison.hernandez@uni.edu.ni \\ 0000-0002-9602-5805 (https://orcid.org/0000-0002-9602-5805)
${ }^{3}$ Universidad Nacional de Ingeniería. Facultad de Arquitectura. Managua, Nicaragua *pablo.espinoza131@std.uni.edu.ni https://orcid.org/0000-0003-4973-8180

(recibido/received: 16-diciembre-2021; aceptado/accepted: 23-noviembre-2021)

\begin{abstract}
RESUMEN
Este documento contiene los resultados del trabajo realizado en el período 2019-2020 como parte de la investigación “ RIESGO URBANO: ENFOQUE DIAGNÓSTICO PARA LA CIUDAD DE MANAGUA " acreditada por la UNI, siendo uno de los objetivos de la misma el diagnóstico de los sistemas de mampostería de bloques de cemento y la propuesta de un nuevo material. En este sentido, a partir de los residuos generados en el laboratorio en las pruebas del sistema de mampostería, se elaboró una propuesta de hormigón reciclado a partir de este escombro, triturando y tamizando estos materiales a granulometría de arena y proponiendo una mezcla de dosificación en base a estudios similares. Los ensayos propuestos, basados en los procedimientos de las normas ASTM y normativas nacionales, fueron el ensayo de compresión de probetas cilíndricas de 15 x $30 \mathrm{~cm}$ y la velocidad del pulso ultrasónico, obteniendo resultados aceptables y útiles para la elaboración de piezas en uso no estructural.
\end{abstract}

Palabras clave: Concreto, agregado reciclado, mampostería de bloque, VPU, prueba de compresión.

\begin{abstract}
This document contains the results of the work carried out in the period 2019-2020 as part of the research " URBAN RISK: DIAGNOSTIC APPROACH FOR THE CITY OF MANAGUA " accredited by the UNI, one of the objectives of the same being the diagnosis of cinder block masonry systems and the proposal of a new material. In this sense, from the waste generated in the laboratory in tests of the masonry system, a proposal for recycled concrete was elaborated from this rubble, grinding and screening these materials to sand granulometry and proposing a dosage mixture based on in similar studies. The proposed tests, based on the procedures of the ASTM standards and national regulations, were the compression test of $15 \times 30 \mathrm{~cm}$
\end{abstract}


cylinder specimens and the speed of the ultrasonic pulse, obtaining acceptable and useful results for the elaboration of non-use parts. structural.

Keywords: Concrete, recycled aggregate, rubble, block masonry, VPU, compressive strength.

\section{INTRODUCCIÓN}

El concreto es uno de los materiales más utilizados en todo el mundo, desde el momento de su concepción y su comercialización. Existen variaciones y diferentes tipos y calidades de estos, según la función y ubicación de los mismos. En la actualidad, la sostenibilidad del planeta y los recursos mismos exigen a la industria de la construcción la búsqueda de nuevas alternativas y el concreto reciclado es una de las opciones más viables. En varios países europeos y en consenso con el tema de los Objetivos de Desarrollo Sostenible, han firmado diferentes acuerdos para la protección de los recursos naturales y la promoción del reciclaje dando luz a materiales alternativos.

Tomando en consideración el constante crecimiento demográfico mundial, que demanda la construcción de infraestructura de vivienda y social, debe conducir a la optimización de la producción o a la búsqueda de materiales cementosos alternativos que resuelvan las necesidades de las generaciones presentes sin comprometer el bienestar de las generaciones futuras. En este contexto surgen los materiales sustentables, formulados a partir de desechos urbanos e industriales, o con materias primas disponibles en la naturaleza sin recurrir a las altas temperaturas de clinquerización (Espinoza 2012).

En 1999, el Parlamento sueco adoptó 15 objetivos de calidad medioambiental, como una herramienta para alcanzar la sostenibilidad en Suecia en una generación. Además, el Parlamento ha adoptado unos 70 objetivos intermedios. Uno de ellos, "Construcciones seguras y sólidas en la sociedad", estipula que la cantidad de residuos destinados al relleno sanitario debe reducirse en un 50\% entre 1994 y 2005 . Este objetivo ambiental también incluye la meta de que los áridos reciclados alcancen un nivel del 15\% de todos los agregados para el año 2010 (Schouenborg 2004).

Además de Europa y estados unidos existen experiencias en el uso de agregados reciclados. En Argentina, por ejemplo, la Universidad de Buenos Aires (UBA) y su Centro Experimental de la Producción (CEP) han realizado investigaciones al respecto, considerando principios entre como son por ejemplo:

- Salud del material: Selección de materiales seguros y saludables. Diseño de productos seguros y saludables para seres humanos y ambiente desde producción a uso y reúso. Ingredientes perpetuamente aprovechados, ciclados y reciclados.

- Reutilización del material: Eliminación del concepto "residuo" a través de un diseño que lo reemplace por "nutriente", biológico (que vuelve a la tierra sin impacto) o tecnológico (que continúa indefinidamente en la cadena productiva) internacionales (aplicables en todo el mundo) para retener el valor completo del nutriente. $100 \%$ de recuperación y posibilidad de reciclado.

Los residuos de construcción y demolición $(\mathrm{C} \& \mathrm{D})$ se pueden utilizar como áridos reciclados para la producción de hormigón. Por lo general, este agregado es una mezcla de desechos de diversos materiales de construcción, especialmente morteros, ladrillos, bloques, virutas de cerámica, concreto, rocas, arena y materiales metálicos, entre otros. En un intento por evaluar la influencia de diferentes composiciones de C\&D sobre las propiedades de los hormigones, se encontró que las variaciones de resistencia al módulo de compresión y deformación, entre otras propiedades, tienen variaciones de como máximo el 10\%, por lo que se concluyó que la variabilidad de C\&D no excluye su uso (Lovato et al., 2012). Esto nos brinda la oportunidad de la re-utilización de estos desechos en la construcción, con capacidades similares y no despreciables para su uso como divisiones o cerramientos, aunque no sea a nivel estructural. 
Un equipo de investigación (Yajnes et al., 2017) que realizó procesos innovadores, en el diseño y la producción de nuevos materiales, fabricados con la inclusión de residuos de la propia locación, reciclados in situ, que aportan capacidad aislante térmica a la envolvente edilicia, (utilizaron $28 \mathrm{~m} 3-12.000 \mathrm{~kg}$ ) de cascotes, medido en su estado de demolición provenientes del caso de estudio (el edificio Olaya de 4 pisos construido), para su reciclado y transformación de residuo en recurso como agregado grueso de hormigones que luego fue utilizado para la construcción de bloques no portantes.

Los materiales reciclados son evaluados con las normativas implementadas para los materiales de construcción de origen natural. Si el área, objeto de estudio, posee sus propias normativas se aplican las disposiciones locales y se deberán considerar normas de carácter internacional como lo son las normas ASTM, ISO. Algunas veces, las normativas representan una limitante (Pérez, 2012). Esto se debe a lo novedoso de estas técnicas y la falta de estandarización y reconocimiento de los comportamientos físicos y mecánicos de los mismos. Sin embargo, hay ensayos que nos brindan una perspectiva del comportamiento de las nuevas propuestas de materiales para la construcción, siempre que estos tengan entre sus materiales constituyentes el cemento. Algunas de estas pruebas y mecanismos técnicos para evaluar las capacidades de los concretos que se utilizan en la construcción están definidos en las normas ASTM C597, ASTM C597, para Nicaragua lo orientado en el RNC-07 y la norma MP-001.

El presente estudio se contiene los resultados obtenidos por medio de ensayos, registrando las propiedades físico - mecánicas de una mezcla de concreto reciclado a partir de escombros de mampostería de boque de cemento, considerando las experiencias antes mencionadas y las normativas correspondientes.

\section{METODOLOGÍA}

\subsection{Materiales}

Se elaboraron muestras de cilindros a partir de la mezcla de concreto estándar o tradicional y también la nueva propuesta de concreto con material reciclado de escombros, siendo los tamaños de estos 15 x $30 \mathrm{~cm}$. Se trabajó con nueve especímenes de concreto reciclado y seis de concreto estándar. Este último se utilizó como comparativo tanto de las características y procedimientos para su contraste final de las características mecánicas de ambas mezclas.

Para este estudio, se entenderá como escombros, aquel material destruido parcial o totalmente, con opción de reciclaje a partir de muros de mampostería de bloques de cemento, los cuales fueron triturados manualmente y posteriormente cribados utilizando la malla \#4 y \#8, de esta manera se obtuvo un árido fino el cual fue sustituido parcialmente del volumen de arena típica utilizada en estos ensayos. Se aprovecharon desechos de materiales (bloques de concreto) que fueron demolidos en el laboratorio y en un periodo de tiempo bastante reciente de las pruebas acá reflejadas.

El diseño de mezclas se realizó acorde a la normativa ASTM C31. La materia prima utilizada fue:

- Árido fino (arena reciclada de escombros).

- Árido Grueso (piedrín de 1/2'”)

- Agua

Los equipos e instrumentos manejados durante el procedimiento:

- Cabezales/cilindros de acero con neopreno

- Aceite (para lubricar cabezales de acero)

- Tamices \#4 y 8 (entre 4 y $2.3 \mathrm{~mm}$ )

- Prensa hidráulica para compresión POWER TEAM Model A, capacidad de 10.000 PSI 
- VPU Controls - Aparato ultrasonido digital E4800

- Cono de Abrams o revenimiento

- Termómetro

Se utilizó proporción de Agua-Cemento (a/c) 0.9. La dosificación fue de 1: 2: 1 (cemento: árido fino: árido grueso), para la cual se sustituyó el 100\% de arena tradicional por arena reciclada. Las mezclas aprobadas fueron sometidas a la prueba de revenimiento usando el cono de Abrams, en condiciones de laboratorio, a temperatura de $34^{\circ} \mathrm{C}$ (ver Tabla 1$)$.

Tabla 1. Proporciones utilizadas en la mezcla

\begin{tabular}{r|rr}
\hline Material & Proporción & Peso en gr \\
\hline Cemento & 1 & 7228.7 \\
Árido & 2 & 19418.4 \\
Fino & & \\
Árido & 1 & 6853.3 \\
Grueso & 0.9 & 6505.83 \\
Agua & 0.9
\end{tabular}

Fuente: Elaboración propia

\subsection{Métodos}

La construcción de los especímenes de concreto se dio atendiendo la rigurosidad normativa, tanto de los reglamentos nacionales y normas internacionales, consideradas desde el momento de elaboración de los cilindros, su proceso de almacenamiento y curado, hasta la aplicación de las pruebas específicas (compresión simple y medición de velocidad ultrasónica):

Esta investigación de corte "Experimento Aplicado", siguió los procedimientos descritos en las siguientes normas:

- Reglamento Nacional de Construcción (RNC-07).

- Norma Mínima De Diseño Y Construcción General De Mampostería MP-001.

- Norma mínima de diseño y construcción de concreto estructural CR-001.

- NMX-C-109-ONNCCE-2013. (cilíndricos con mortero de azufre de 15x30).

- ASTM C31 - Standard Practice for Making and Curing Concrete Test Specimens in the Field

- ASTM C39 - Standard Test Method for Compressive Strength of Cylindrical Concrete Specimens

- ASTM C597 - Standard Test Method for Pulse Velocity Through Concrete.

Los procedimientos se realizaron en su totalidad en el laboratorio de Pruebas mecánicas de la Facultad de Tecnología de la Construcción, de conformidad a las etapas definidas para esta investigación:

\section{- Primera etapa: Selección y adquisición de materia prima}

Se definieron los materiales y luego de la revisión documental se procedió a proponer un posible dosificación, misma que sería ajustada (relación a/c) de considerarlo necesario. Los materiales utilizados fueron cemento (Cemex), arena (Motastepe), material reciclado (triturado y tamizado a partir de escombros)

- Segunda etapa: Elaboración y curado de los especímenes o muestras cilindros de concreto Se elaboraron dos tipos de especímenes paralelamente, uno con mezcla de concreto estándar (6 cilindros) y otras nueve muestras con agregado de material reciclado. Estos procedimientos fueron realizados en laboratorio, acorde a las normativas NMX-C-109-ONNCCE-2013 y las ASTM C31. 
El curado de los cilindros se realizó sumergidos estos en una mezcla con relación de 45 gr de Hidróxido de Calcio por cada $15 \mathrm{~kg}$ de agua.

\section{- Tercera etapa: Ensayos acorde las normativas MP-001, ASTM C39 y ASTM C597}

De los nueve cilindros creados con la mezcla de concreto reciclado, 6 fueron sometidos a prueba de compresión simple y velocidad de pulso ultrasónico, los otros tres se resguardaron como testigos (RC1, RC6, RC9). Las pruebas de compresión se realizaron en dos momentos (28 y 56 días), en condiciones de laboratorio, por medio del equipo POWER TEAM Model A-10.000 PSI. El procedimiento seguido es el descrito en la ASTM C39.

Los ensayos no destructivos constituyen hoy en día una herramienta muy útil para determinar la calidad del concreto endurecido. Entre una gama variada de estos, el ultrasonido o método de medición de pulso ultrasónico ha sido utilizado con éxito desde hace más de 50 años en muchos países. Es un método que día a día ha ido incrementando sus aplicaciones en el campo de la tecnología del concreto, como una alternativa de ensayo no destructivo (VIdaud y Vidaud 2016). En este sentido, se utilizó un equipo especializado (VPU Controls E4800) para medir conforme lo establecido en la ASTM C597.

El VPU consiste en una toma de medidas del tiempo que demora un pulso ultrasónico (frecuencia entre 20 y $150 \mathrm{kHz}$ ) al recorrer la distancia entre un transductor emisor Tx y un transductor receptor Rx, ambos acoplados al concreto en estudio (VIdaud y Vidaud 2016). Este tipo de pruebas con un alto grado de fiabilidad pueden ser muy efectivas especialmente en evaluaciones de concretos muy antiguos edificios con valor patrimonial o inclusive edificios y conjuntos habitacionales para habitantes con escasos recursos.

\section{Los reciclados de la construcción}

\section{DESARROLLO}

Los materiales reciclados son evaluados con las normativas implementadas para los materiales de construcción de origen natural. Si el área, objeto de estudio, posee sus propias normativas se aplican las disposiciones locales y se deberán considerar normas de carácter internacional como lo son las normas ASTM, ISO. Algunas veces, las normativas representan una limitante (Pérez, 2012). Esto se debe a lo novedoso de estas técnicas y la falta de estandarización y reconocimiento de los comportamientos físicos y mecánicos de los mismos.

Generalmente producto de las demoliciones intencionales, o de la ocurrencia de eventos catastróficos que destruyen total o parcialmente edificios en las ciudades, la generación de desechos es una constante en todo el mundo. Sin embargo, estos desechos nos brindan una oportunidad, al aplicarles diferentes procesos mecánicos o químicos que les dan una segunda oportunidad de uso en las mezclas para producir nuevos materiales, ya sea como morteros base para bloques u otro material de este tipo, para concretos o incluso materiales de sub-base en infraestructura vial.

El empleo de agregados reciclados para la fabricación de concreto tiene su origen en los años posteriores a la Segunda Guerra Mundial en Alemania; período en que los escombros provenientes de los bombardeos, se encontraban disponibles en grandes volúmenes en muchas partes de Europa (Vidaud y Vidaud 2015).

En general la utilización de concreto elaborado con agregado de ladrillo reciclado es aun escasa en proporción con el concreto y hormigón tradicional; sin embargo, en varios países se han llevado a cabo proyectos ingenieriles, algunos se resumen a continuación (Schouenborg, B., Aurstad, J., \& Pétursson, P. 2004):

- Holanda: desde 1994 se ha venido implementando el uso de este tipo de materiales en hormigón estructural, con un remplazo máximo del $20 \%$ del agregado grueso. Han llevado a cabo varios 
proyectos piloto donde se ha remplazado el $20 \%$ del agregado grueso en la construcción de un viaducto cerca de la ciudad de Helmond por una mezcla de concreto y ladrillo triturado. En las obras de la compuerta del puerto de Schijndel, se emplearon alrededor de $300 \mathrm{~m} 3$ de concreto con agregado reciclado procedente de una mezcla de hormigón y ladrillo.

- Alemania: el uso de agregado reciclado data desde 1950 y se ha usado principalmente para la Construcción de viviendas. En 1996 y 1997, se desmanteló una zona militar a las afueras de Itzehoe que produjo aproximadamente unas 50000 toneladas de escombros cerámicos, los cuales se utilizaron para la edificación nuevamente de esa misma zona.

- Reino Unido: en Cardington se utilizó este tipo de material, para la construcción de la losa de la segunda planta de un edificio de esta ciudad. La armada y de $50 \mathrm{~cm}$ de espesor, utilizó sustitución del 20\% del agregado grueso y se emplearon unas 100 toneladas de agregado reciclado, obteniendo cercanas a los $60 \mathrm{~N} / \mathrm{mm} 2$ a los 91 días.

- Estados Unidos: de los aproximadamente 2,7 millones de toneladas anuales de agregado reciclado se utiliza actualmente de 10 a $15 \%$ para pavimentación, de 20 a 30\% para construcción de carreteras y otros trabajos de mantenimiento y al rededor del 60 a $70 \%$ se utilizan para la elaboración de concreto estructural.

- Japón: aunque Japón ha investigado por más de un cuarto de siglo sobre la reutilización de agregados de demolición para la elaboración de concreto, este reciclado no ha sido posible en su totalidad, debido a que este material no cumple a cabalidad con la norma JIS A-5308, que establece las especificaciones de concreto premezclado. Sin embargo, en la actualidad este material se está utilizando en un $96 \%$ como subbase en la construcción de carreteras.

Varios estudios indican la viabilidad de esta alternativa, que depende del porcentaje de reposición empleado. Sin embargo, no existe consenso sobre el comportamiento de los hormigones producidos con este material. Esto se debe a la alta variabilidad de las composiciones, tipo de equipo de trituración, porcentajes de reemplazo, y si se hizo prehumedecimiento o no, uso de aditivos o no, tipo de cemento y otros factores (Lovato et al., 2012).

\section{Impacto por la utilización de concretos reciclados}

Con respecto a la calidad técnica y profesional del sector construcción en Nicaragua,si bien es cierto, existen ofertas a nivel técnico y de pregrado para la formación de maestros de obra, ingenieros civiles y arquitectos en diferentes instituciones públicas y privadas; a nivel de posgrado no existen programas orientados a la investigación de materiales, y de manera específica de "materiales de construcción". La ausencia de laboratorios de materiales bien equipados para la evaluación y el mejoramiento de materiales, institutos, centros de investigación, así como de la infraestructura científica adecuada, es otra de las carencias presentes en el país (Hernández 2017).

Los agregados reciclados (AR) se manejan en la literatura especializada según su origen en: procedentes de concreto, cerámicos y mixtos. Muchas normativas hoy no permiten el uso de estos dos últimos tipos en concreto estructural, otras admiten el empleo de los cerámicos solo en concretos no estructurales, y otras restringen este uso solo con agregados procedentes de concretos. Si bien es cierto que hoy resultan muy interesantes las expectativas de crecimiento del empleo de estos AR para la fabricación de concreto; también lo es que se han dejado abiertas muchas brechas para el estudio y la investigación acerca del comportamiento de estos concretos sustentables (Vidaud y Vidaud 2015).

En general, este tipo de materiales brindan una gran cantidad de ventajas, principalmente desde el punto de vista económico y de reducción de impactos negativos en el medio natural. Algunas de estas ventajas son (Rivera et al., 2020): 
- Generación de múltiples trabajos de investigación, tanto a nivel de pregrado y posgrado, que incluye el capital humano de las universidades, instituciones gubernamentales y empresa privada (innovación).

- Integrar el reciclaje de los desperdicios de construcción dentro de la planificación del ciclo de vida del edificio, permite un manejo más coherente y amigable para la reducción de impactos negativos al entorno natural.

- Utilizar estos materiales ya sea en granulometrías bajas o medias, incluso a nivel de partículas más finas como polvo, permite un ahorro en el consumo de otros materiales y dentro de los requisitos de la energía de procesos (Process Energy Requirement - PER), generando una eficiencia del proceso de fabricación individual (que se implica ganancias en los aspectos de los combustibles que se utilizan en la fabricación de los materiales, la relación materiales vs distancias, etc.).

- Se abren puertas para otro tipo de mercado de construcción de materiales reciclados, que podría dinamizar la industria de la construcción. Con la inversión y tratamiento adecuado, los productos resultantes de estos procesos podrían generar ganancias económicas a quienes se involucren en estas actividades.

\section{Tipos de desperdicios y escombros generados en Managua}

Regularmente encontramos en las ciudades con mayor actividad económica una dinámica más frecuente de remodelaciones, ampliaciones y nuevas construcciones, siendo estos procesos los orígenes para grandes volúmenes de desperdicios y escombros.

De acuerdo con la Cámara Nicaragüense de la Construcción - CNC, durante el 2017 hubo gran dinamismo del sector, debido principalmente a la fuerte inversión privada y pública que desarrollan diversos proyectos en el país. Entre los cuales tenemos: el nuevo estadio nacional de béisbol "Dennis Martínez", el polideportivo "Alexis Argüello", los pasos a desnivel de las "Piedrecitas y Siete sur", la prolongación de la "Pista Larreynaga", entre otros. La formulación y ejecución de estos proyectos es visible en Managua. Los esfuerzos de inversión están orientados hacia lograr la modernidad de la capital, al ordenamiento y a satisfacer las necesidades de la población. Sin embargo, es necesario considerar construir para el futuro "obras durables", que tengan una larga vida de servicio y resistan a las condiciones ambientales a las que están expuestas (Hernández 2017). Paralelamente que se desarrollan muchos nuevos edificios e infraestructuras, estos son en muchos casos ubicados en los sitios de otros, generando en sí mismos y su origen, nuevos desechos para la ciudad.

En la ciudad de Managua uno de los productos más utilizados en la construcción es el bloque de mortero de cemento, siendo este uno de los principales productos de escombros y desechos frecuentes, lo que genera una oportunidad y una justificación de la necesidad de este tipo de estudios.

\section{RESULTADOS Y ANÁLISIS}

De acuerdo a las normativas rectoras en Nicaragua (RNC-07) que se fundamentan en normativas internacionales, se aceptan como concretos para el trabajo en el país los concretos: concreto estructural, concreto liviano (resistencia mínima de entre 1.440 y $1.840 \mathrm{~kg} / \mathrm{m} 3$ ), concreto presforzado y concreto prefabricado. Para aplicaciones con concreto normal, o liviano, de estructuras de concreto estructural, en general se limita el valor mínimo del fcâ a $210 \mathrm{~kg} / \mathrm{cm} 2$ y no se establece valor máximo para el mismo. En el caso aplicaciones con concreto liviano para aplicaciones de marcos resistentes a momento y muros estructurales del tipo especial igualmente se limita el valor mínimo del fcâ a $210 \mathrm{~kg} / \mathrm{cm} 2$ pero adicionalmente se establece un valor máximo de $350 \mathrm{~kg} / \mathrm{cm} 2$ (RNC-07). 


\section{Ensayos a compresión}

La ejecución de mezclas de prueba en laboratorio, estarán de acuerdo con ASTM C-39 en cilindros preparados según ASTM C-192. Si no se tiene registro de las instalaciones para la producción de concreto basado en 30 pruebas de resistencia consecutivas que representen materiales y condiciones similares a los esperados, la resistencia a la compresión promedio requerida deberá ser $85 \mathrm{Kg} / \mathrm{cm}^{2}$ superior a la f'c. Cada punto deberá representar el promedio de por lo menos 3 cilindros probados a los 28 días o a edades menores según lo especificado en los planos de diseño o especificaciones. (RNC - 07).

La resistencia a la compresión de las mezclas de concreto se obtuvo siguiendo el procedimiento establecido en la norma ASTM C 39 (ver Figura 1). Se destinaron a este ensayo 6 muestras, de la cuales las RC-5 y RC8 que estaban previstas originalmente para ser probadas a los 56 días, fueron sometidas a la compresión más tarde por efectos de bioseguridad en la operatividad del laboratorio, como medida de precaución del tema pandemia. La muestra RC-7 fue eliminada debido a comportamiento discrepante al ser sometida al ensayo. Las resistencias obtenidas se presentan en la Tabla 2.
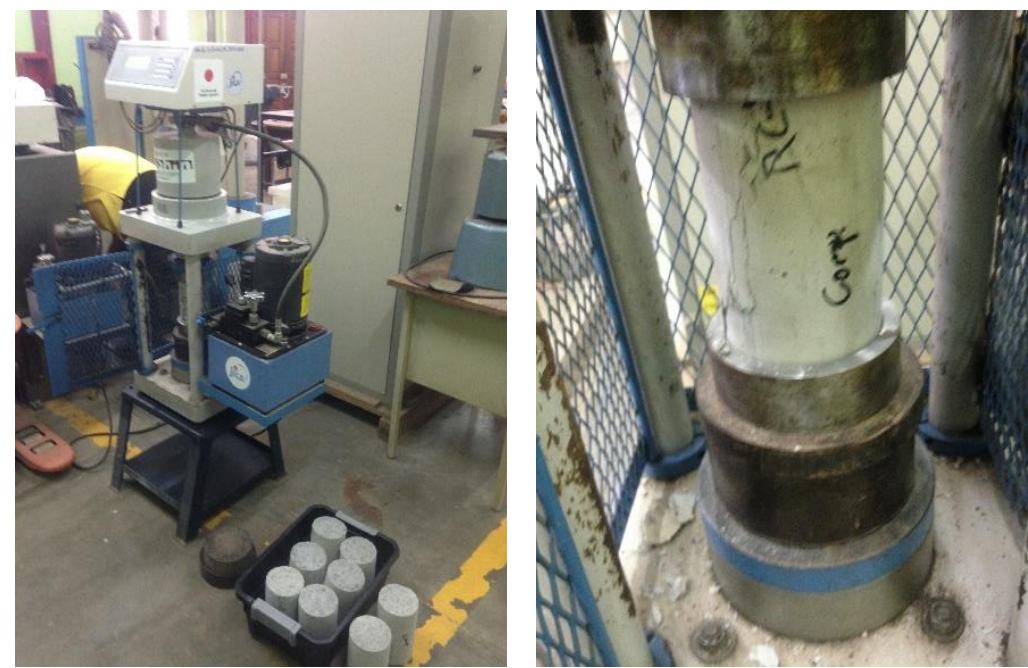

Figura 1: Ensayos a compresión de especímenes; Izq. Prensa hidráulica POWER TEAM, Der. Cilindro ensayado Fuente: Foto de los autores

Tabla 2. Codificación y resistencia a la compresión

\begin{tabular}{l|lll}
\hline $\begin{array}{c}\text { Código del } \\
\text { espécimen }\end{array}$ & Edad (días) & $\begin{array}{c}\text { Resistencia a la } \\
\text { compresión (Lbf) }\end{array}$ & $\begin{array}{c}\text { Resistencia a la } \\
\text { compresión } \mathbf{( K g / c m 2})\end{array}$ \\
\hline RC-2 & 28 & 35.000 & 188.52 \\
RC-3 & 28 & 35.110 & 171.70 \\
RC-4 & 28 & 31.900 & 190.80 \\
RC-5 & 280 & 37.030 & 199.46 \\
RC-8 & 280 & 37.560 & 202.17 \\
\hline
\end{tabular}

Fuente: Elaboración propia.

En investigaciones similares (Martinez y Mendoza 2006) podemos observar que el comportamiento a la compresión en los ensayos (a 28 días) se mantiene entre los 188 y $360 \mathrm{Kg} / \mathrm{cm}$ incrementándose su valor en relaciones a/c bajas (0.56-0.68), se observa que los concretos reciclados tienen mayores resistencias de lo esperado respecto de los naturales para una misma relación a/c. Hay que tomar en cuenta que los concretos reciclados requieren de mayores consumos de agua que los naturales para alcanzar revenimientos similares. Por otro lado, otros trabajos sugieren que relaciones menores de 1 y de 0.5 o un poco más pueden ser funcionales si el porcentaje de agregado en la mezcla es menor del 25\%. Este trabajo dado que se utilizó 
una proporción mayor de agregado fino reciclado (40\%), necesitó más agua para poder aglomerar eficientemente con el piedrín, el cemento y el agua, misma razón por la cual se incrementó la relación a/c.

De acuerdo a estudios similares, podemos identificar comportamientos a la compresión análogos, a pesar la diferencia en los porcentajes de material reciclado utilizado y agua. El valor máximo obtenido a los 28 días fue de $190.8 \mathrm{~kg} / \mathrm{cm} 2$ manteniendo valores constantes en el muestreo. Los resultados de los ensayos a los especímenes RC-5 y RC-8 demuestran un ligero incremento en las capacidades de ambos. A continuación se establece una comparación de estos resultados elaborados por los autores, en relación a tres tipos de concretos reciclados (incluyendo el de elaboración propia) y el concreto estándar elaborado paralelamente, ver Tabla 3.

Tabla 3. Comparación de ensayos a compresión de diferentes concretos reciclados

\begin{tabular}{|c|c|c|c|c|c|c|c|c|c|c|c|}
\hline \multirow[t]{2}{*}{$\begin{array}{l}\text { Fc } \\
\text { (28 Días) }\end{array}$} & \multicolumn{3}{|c|}{$\begin{array}{l}\text { Martinez } \\
\text { Mendoza } 2006\end{array}$} & $\begin{array}{l}\text { Lovato } \\
2012\end{array}$ & et al., & \multicolumn{2}{|c|}{$\begin{array}{l}\text { Valverde } \\
\text { Castellanos } \\
2017\end{array}$} & \multicolumn{2}{|c|}{ Ensayos CR } & \multicolumn{2}{|c|}{ Ensayos CS* } \\
\hline & $\begin{array}{l}\text { Min. } \\
185.53\end{array}$ & $\begin{array}{l}\text { Max. } \\
385.61\end{array}$ & & $\begin{array}{l}\text { Min. } \\
132.56\end{array}$ & $\begin{array}{l}\text { Max. } \\
330.3 \\
9\end{array}$ & $\begin{array}{l}\text { Min. } \\
143.78\end{array}$ & $\begin{array}{l}\text { Max. } \\
257.9 \\
9\end{array}$ & $\begin{array}{l}\text { Min. } \\
171.70\end{array}$ & $\begin{array}{l}\text { Max. } \\
190.8 \\
0\end{array}$ & $\begin{array}{l}\text { Min. } \\
302.34\end{array}$ & $\begin{array}{l}\text { Max. } \\
372.1 \\
4\end{array}$ \\
\hline Mediana & \multicolumn{3}{|c|}{303.80} & \multicolumn{2}{|c|}{166.21} & \multicolumn{2}{|c|}{199.86} & \multicolumn{2}{|c|}{188.52} & \multicolumn{2}{|c|}{352.21} \\
\hline $\begin{array}{l}\text { Promedi } \\
\text { o }\end{array}$ & \multicolumn{2}{|c|}{290.16} & & \multicolumn{2}{|c|}{187.22} & \multicolumn{2}{|c|}{196.91} & \multicolumn{2}{|c|}{183.68} & \multicolumn{2}{|c|}{347.43} \\
\hline
\end{tabular}

Fuente: Elaboración propia.

La afirmación de que la resistencia de los concretos convencionales aumenta con la disminución de la a/c, no podría ser aplicada "a priori" en el caso de los concretos reciclados. En estos la resistencia también depende de la calidad del material utilizado. Siguiendo esta idea, a partir de un árido reciclado de baja calidad (concreto que de origen fue de baja resistencia) se obtendrá un concreto reciclado con un nivel de resistencia que no podrá ser superado aunque se disminuya la relación a/c; sin embargo, si el árido reciclado es de alta calidad (concreto de origen de buena resistencia y buen estado), los concretos reciclados resultantes pueden alcanzar resistencias cuya correspondencia con la a/c es similar a la de los concretos convencionales (Vidaud y Vidaud 2015). En el caso de este estudio, los áridos reciclados son producto de la mezcla de escombros 2 tipos de bloques de morteros, siendo uno de ellos de alta calidad y otra inferior, las cuales se mezclaron hasta obtener un material más homogéneo.

Hay que destacar que esta práctica genera beneficios ambientales y debe considerarse desde esta perspectiva. Para Lovato et al., 2012 los resultados también muestran que cuando se sustituyen contenidos de áridos naturales por áridos mixtos (naturales con reciclados) hasta el 50\% los, hormigones producidos son técnicamente viables, y es posible producir hormigones con una resistencia de unos $25 \mathrm{MPa}$, (254.93 $\mathrm{kgf} / \mathrm{cm}^{2}$ ) con una buena mecánica y rendimiento de durabilidad.

Este factor del agua y la humedad para los áridos reciclados es importante, pudiendo generar notorios cambios en los rendimientos y consumos de cemento. En la experiencia de Lovato et al., 2012, se tuvo en cuenta la absorción de agua de los áridos prehumedeciéndolos $15 \mathrm{~min}$ antes del proceso de mezcla. Otros autores destacan sin embargo la necesidad de prehumedecimiento de los áridos; no hay ninguna recomendación sobre la duración de este procedimiento. Este procedimiento tiene como objetivo compensar parcialmente la absorción de material, evitando que el agua de mezcla sea absorbida por el agregado. El agregado fino recibió el 55\% de la absorción total mientras que, para el agregado grueso, se utilizó el 70\% de la tasa de absorción total. Después de las relaciones agua / cemento iniciales (a / c inicial) se añadió agua 
a la mezcla hasta que alcanzó el asentamiento definido de $100 \pm 20$ mm, lo que resultó en un nuevo a / c (a / c real) para cada mezcla.

\section{Prueba de Velocidad de Pulso Ultrasónico - VPU}

El método de ultrasonido se basa entonces en la teoría de la propagación de las ondas compresionales en un medio infinito, homogéneo, isotrópico y elástico. De acuerdo a esta teoría física, la velocidad de las ondas depende principalmente de la densidad y de las características elásticas del medio que recorren. Respecto al uso de este método del VPU, Varios autores consideran que aunque este ensayo no permita evaluar directamente la resistencia del concreto, correlacionando las medidas ultrasónicas con la resistencia a partir de una combinación del método con calibración en laboratorio, podría ofrecerse una estimación de la resistencia característica del concreto ensayado con una precisión de $\pm 20 \%$ (VIdaud y Vidaud 2016).

El estudio de VPU se aplicó constantemente a 6 de los especímenes construidos (ver Figura 2). Se observa una reducción en la velocidad de la velocidad de pulso ultrasónico a mayor edad en cada mezcla de concreto. Este decrecimiento se mantiene constante en un rango de $3-1 \%$. Estudios muestran una tendencia a reducir el valor de esta medición en la mezclas con mayor contenido de agregado de concreto reciclado. Por último, la velocidad de pulso ultrasónico disminuyó en las mezclas con mayor relación agua-cemento (Laverde 2017).

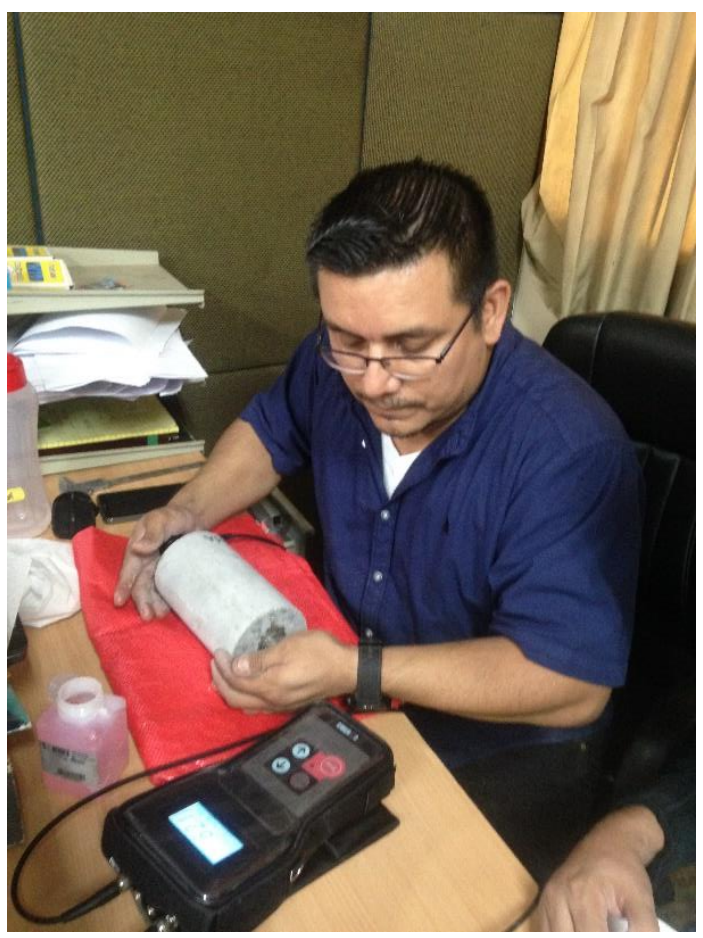

Figura 2: Medición de velocidad ultrasónica en cilindros de concreto reciclado Fuente: Foto de los autores

La aplicación de pruebas de pulso ultrasónico se realizó en 3 fechas a 6 especímenes con agregado reciclado (ver Tabla 4), siendo los siguientes los valores obtenidos:

Tabla 4. Velocidades de Pulso ultrasónico en especímenes

\begin{tabular}{|c|c|c|c|c|}
\hline & Espécimen & $\begin{array}{l}\mathrm{V}(\mathrm{D} / \mathrm{T}) \\
28 \text { días }\end{array}$ & $\begin{array}{l}\mathrm{V}(\mathrm{D} / \mathrm{T}) \\
56 \text { días }\end{array}$ & $\begin{array}{l}\text { V (D/T) } \\
84 \text { días }\end{array}$ \\
\hline $\begin{array}{l}\text { Concreto } \\
\text { reciclado }\end{array}$ & $\begin{array}{l}\mathrm{RC} 2 \\
\mathrm{RC} 3\end{array}$ & $\begin{array}{l}3101.10 \\
3105.59\end{array}$ & --- & \\
\hline
\end{tabular}




\begin{tabular}{|c|c|c|c|c|}
\hline & Espécimen & $\begin{array}{l}\mathrm{V}(\mathrm{D} / \mathrm{T}) \\
28 \text { días }\end{array}$ & $\begin{array}{l}\mathrm{V}(\mathrm{D} / \mathrm{T}) \\
56 \text { días }\end{array}$ & $\begin{array}{l}\text { V (D/T) } \\
84 \text { días }\end{array}$ \\
\hline \multirow{10}{*}{$\begin{array}{l}\text { Concreto } \\
\text { estándar }\end{array}$} & RC4 & 3025.98 & --- & \\
\hline & $\mathrm{RC} 5$ & 3283.36 & 3092.35 & 3157.89 \\
\hline & $\mathrm{RC7}$ & 3111.79 & 3080.13 & 3038.82 \\
\hline & $\mathrm{RC} 8$ & 3118.34 & 3094.28 & --- \\
\hline & $\mathrm{M} 1 / 2 \mathrm{~A} 3$ & 3458.31 & & \\
\hline & M1/2A5 & 3464.93 & & \\
\hline & M1/2A6 & 3404.40 & & \\
\hline & M1/2B5 & 3441.31 & & \\
\hline & $\mathrm{M} 1 / 2 \mathrm{~B} 7$ & 3486.48 & & \\
\hline & $\mathrm{M} 1 / 2 \mathrm{~B} 8$ & 3435.36 & & \\
\hline
\end{tabular}

Fuente: Elaboración propia.

Para las mezclas de uso normal que están entre $21 \mathrm{MPa}$ y $35 \mathrm{MPa}$, se considera como óptimo un desempeño que se ubique en el rango de $3660 \mathrm{~m} / \mathrm{s}$ y $4575 \mathrm{~m} / \mathrm{s}$ (Bedoya y Dzul 2015). Sin embargo, muestras que oscilan entre los rangos de 3050 y 3660 es un concreto Cuestionable, pero no malo (VIdaud y Vidaud 2016). Como se observa en la Tabla 5, para valores obtenidos a los 28 días en relación con los resultados de otros estudios, estadísticamente no marcan una discrepancia demasiado distante en sus comportamientos. La muestra de mezcla elaborada por los autores, similar a la de Bedoya y Dzul 2015 que también utilizó 100\% de reemplazo, se acerca a los valores de velocidad en un $75 \%$. En contraposición, el ensayo reciclado tiene una velocidad de un $90 \%$ de la mezcla de referencia de concreto estándar.

Tabla 5. Comparación de VPU en concreto reciclados

\begin{tabular}{c|cccc}
\hline VPU & $\begin{array}{c}\text { Bedoya y Dzul } \\
\mathbf{2 0 1 5}(\mathbf{5 0 \%} \\
\text { reemplazo) }\end{array}$ & $\begin{array}{c}\text { Bedoya y Dzul } \\
\mathbf{2 0 1 5}(\mathbf{1 0 0 \%} \\
\text { reemplazo) }\end{array}$ & Ensayos CR & Ensayos CS \\
\hline $\begin{array}{c}\text { Promedios } \\
\text { 28 días }\end{array}$ & 4437.5 & 4134.5 & 3077.56 & 3448.47 \\
\hline
\end{tabular}

Fuente: Elaboración propia.

\section{CONCLUSIONES}

Las diferencias en materiales y procedimientos experimentales dificultan la comparación de los resultados de los diversos estudios y muestran la necesidad de estandarizar procedimientos y métodos (Lovato et al., 2012). Sin embargo, la existencia de diferentes experiencias similares difundidas en artículos y congresos, permite construir mejor estos procedimientos e identificar valores que alcanzar para buenos concretos reciclados.

El aprovechamiento de estos residuos constituye un aspecto importante a nivel mundial ya que esto representa un beneficio económico, debido a la incorporación de materiales de desecho al proceso de construcción y a su vez ayuda a la preservación de los recursos naturales (Pérez 2012).

Se hace necesario para futuras pruebas aplicar otro paquete de ensayos a las muestras, por ejemplo, Lovato et al., 2012 sugiere ensayos de absorción de agua que se obtiene por inmersión, considerando las siguientes etapas: secado de la muestra en un horno durante $72 \mathrm{~h}$ y determinación de su materia en estado seco; saturación de la muestra en agua durante $72 \mathrm{~h}$; mantenimiento de la muestra en agua hirviendo durante $5 \mathrm{~h}$; y determinación de su materia en estado saturado, luego de remover el exceso de agua con un paño húmedo. 
Para la resistencia a la compresión, el mayor contenido de agregado reciclado tiende a reducir la resistencia de los hormigones, teniendo el agregado grueso una mayor influencia negativa en esta propiedad. Como el agregado reciclado es más frágil que el agregado natural, las grietas tienden a propagarse también en los agregados, reduciendo la resistencia a la tracción (Lovato et al., 2012).

Para los especímenes construidos en esta investigación se requirió un incremento de las relaciones en experimentos similares (Martinez y Mendoza 2006, Lovato et al., 2012 y Valverde y Castellanos 2017) en lo referente a la proporción de agua/cemento y comparado con una mezcla regular de concreto estándar. Esto debido también a que se realizó una sustitución del total del árido fino estándar por el árido reciclado, lo que ocasionó mayor necesidad de agua para aglomerarse con el piedrín y cemento. Sin embargo, a pesar que los resultados fueron inferiores (sin generar grandes distancias), considerando la dosificación ensayada, se comprueba la importancia del uso de estos materiales reciclados.

Desde el punto de vista ambiental estos hormigones permiten una reducción del $50 \%$ en el consumo de áridos naturales finos y gruesos, lo que contribuye positivamente a la sostenibilidad de esta zona. Además se ha demostrado que para el mismo nivel de resistencia a la compresión axial, superior a $20 \mathrm{MPa}$, a pesar del mayor consumo de cemento, los costes son similares a los del hormigón de referencia (mezclas estándares). Para niveles de resistencia más bajos, los hormigones producidos con residuos de construcción y demolición reducen los costos de producción de hormigón en aproximadamente un 20\% (Lovato et al., 2012).

Se comprueba que existe una relación directa entre la relación Agua - Cemento $(\mathrm{a} / \mathrm{c})$ y la dosificación utilizada, en donde relaciones menores a 1 generalmente incrementan las capacidades de resistencia de los concretos reciclados. De igual manera, el comportamiento de los especímenes ante la prueba de pulso ultrasónico, brindo resultados prometedores, si bien ligeramente inferiores a otros similares, se mantienen en márgenes superiores a concretos de mala o deficiente calidad.

\section{AGRADECIMIENTOS}

Este trabajo fue realizado en el marco de los proyectos de investigación e innovación 2019-2020, impulsados por la Vice - rectoría de Investigación de la Universidad Nacional de Ingeniería. Especial agradecimientos al Dr. Oscar Gutiérrez, Decano de la Facultad de Tecnología de la Construcción, por su gestión para el uso del laboratorio de pruebas a materiales y al equipo de investigadores nóveles, principalmente la br. Rachel Guerrero.

\section{REFERENCIAS}

Bedoya, C., \& Dzul, L. (2015). El concreto con agregados reciclados como proyecto de sostenibilidad urbana. Ingeniería de Construcción, 99-108.

Beltrán, G. G., \& Sandí, A. M. (2011). Recomendaciones para obtener resultados confiables de resistencia de cilindros de concreto. Métodos y materiales, 1(1), 4-13.

Hernández, E. (2017). Construcción durable-Una visión de futuro para Nicaragua. Revista Arquitectura+, 2(4), 8-12.

Lovato, P. S., Possan, E., Dal Molin, D. C. C., Masuero, Â. B., \& Ribeiro, J. L. D. (2012). Modeling of mechanical properties and durability of recycled aggregate concretes. Construction and Building Materials, 26(1), 437-447. 
Martínez-Soto, I. E., \& Mendoza-Escobedo, C. J. (2006). Comportamiento mecánico de concreto fabricado con agregados reciclados. Ingeniería, investigación y tecnología, 7(3), 151-164.

Pérez, Á. (2012). Uso del triturado del ladrillo reciclado como agregado grueso en la elaboración de concreto. INGENIUM, 116-125.

Rivera, E., Guerrero, R., Espinoza, P., Millon, G., \& Areas, E. (2020). Concretos reciclados, posibilidades de investigación desde el pregrado. Revista Arquitectura+, 9(5), 28-39. https://doi.org/10.5377/arquitectura.v9i5.9918

Yajnes, M. E., Caruso, S. I., Kozak, D., Kozak, A., \& Mühlmann, S. (2017). Waste management and block production of recycled material in situ in a building of intermediate scale in the city of Buenos Aires, Argentina. In Proceedings of the 3rd International Congress on Sustainable Construction and Eco-Efficient Solutions (2017), p 1035-1058. Universidad de Sevilla. Escuela Técnica Superior de Arquitectura.

Valverde, J. A. V., \& Castellanos, N. T. (2017). Propiedades mecánicas, eléctricas y de durabilidad de concretos con agregados reciclados. Revista de la Escuela Colombiana de Ingeniería, (108).

Vidaud, E., \& Vidaud, I. (2016). Ultrasonido: Aplicación para la determinación del módulo de elasticidad dinámico. Construcción y tecnología en concreto, 31-35.

Vidaud, I. N., \& Vidaud, I. (2015). Propiedades físico-mecánicas de los concretos reciclados. Construcción y Tecnología en Concreto, 26.

\section{SEMBLANZA DE LOS AUTORES}
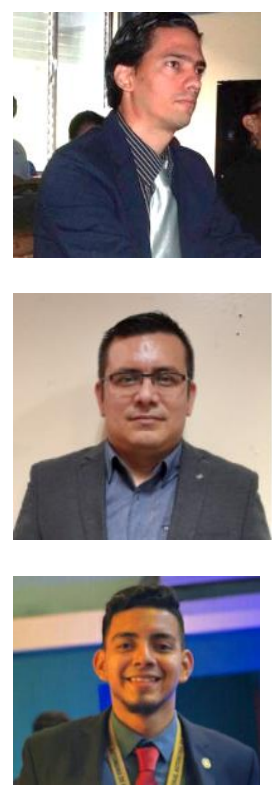

Erasmo José Aguilar Arriola: Obtuvo el grado de Arquitecto en la Universidad Nacional de Ingeniería, Nicaragua, donde actualmente es profesor auxiliar, investigador acreditado períodos 2016-2017 y 2019-2020, editor fundador de la Revista Arquitectura +. Estudios de maestría en Evaluación de Riesgos y Reducción de Desastres por el Instituto de Geología y Geofísica (IGG CIGEO) de la UNAN-Managua. Trabajos de investigación en teoría de la arquitectura, vivienda urbana y mampostería a partir de bloques de cemento.

\section{Eddisson Francisco Hernández}

Obtuvo el grado de Arquitecto en la Universidad Nacional de Ingeniería, Nicaragua, Grado de doctor en el instituto Politécnico de México. Actualmente es profesor auxiliar, investigador acreditado períodos 2019-2020, 2020-2021. Múltiples participaciones en congresos y eventos científicos, y publicaciones en revistas de ciencia y arquitectura.

\section{Pablo Antonio Espinoza}

Egresado de la carrera de arquitectura el año 2020. Investigador novel desde 2019 en la línea de materiales a base de cemento y urbanismo. Participación en ferias científicas de la UNI. Actualmente forma parte del equipo de investigación que desarrolla el tema de "Patrones de Ruralidad y Metropolización en la Región Metropolitana de Managua". 